\title{
Betreuung von Tumorpatienten
}

\section{Neues Internetportal für onkologische Pflegekräfte: www.pflege-onkologie.de}

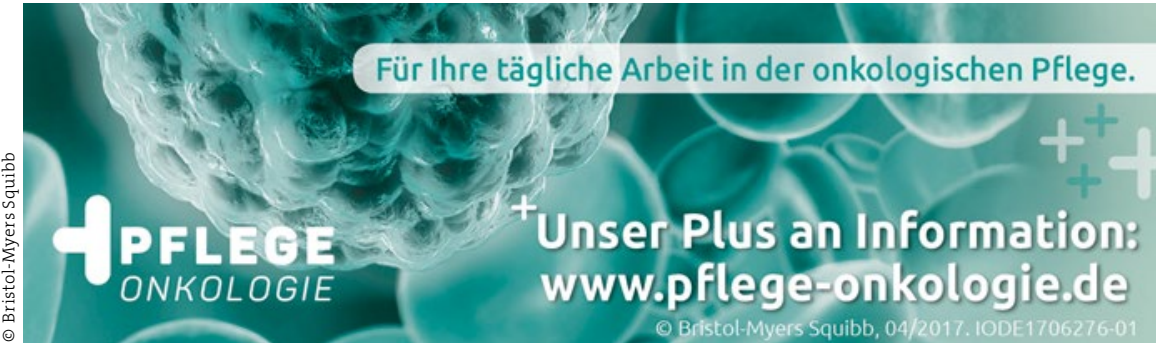

") Mit der Immunonkologie konnte das herkömmliche und etablierte spektrum der Tumorbehandlungen Operation, Bestrahlung, Chemo- und zielgerichtete Therapien in jüngster Zeit um eine weitere effektive Option erweitert werden (Borghaei H et al.; Eur J Pharmacol 2009; 625: 41-54). Immunonkologische Konzepte nutzen die natürliche Fähigkeit des körpereigenen Abwehrsystems, Tumore $\mathrm{zu}$ erkennen, $\mathrm{zu}$ bekämpfen und zu zerstören. Wie bei den anderen Behandlungsmaßnahmen auch, ist vor Beginn einer immunonkologischen Therapie ein ausführliches Patientengespräch unverzichtbar, um über die Möglichkeiten dieser Strategie, eventuelle Nebenwirkungen und deren Management aufzuklären.
Im oftmals hektischen Klinikalltag haben Pflegekräfte häufig einen intensiveren Kontakt zum Patienten als Ärzte und sind für viele Probleme oft die ersten Ansprechpartner. Um das Pflegepersonal bei dieser anspruchsvollen Aufgabe zu unterstützen, bietet das Unternehmen Bristol-Myers Squibb das speziell für Pflegekräfte entwickelte, neue Internet-Portal www.pflegeonkologie.de an. Die Website greift ein breites Spektrum an Themen auf, die im pflegerischen Alltag wichtig sind. So liefert sie Informationen $\mathrm{zu}$ mehr als 20 Tumorindikationen und zu den verschiedenen Behandlungsoptionen in der Krebsmedizin. Nebenwirkungen und Supportivmedizin, Möglichkeiten der psychoonkologischen Unterstüt- zung und die bei Tumorpatienten wichtige Ernährung werden angesprochen. Außerdem informiert die Website mit einem Kongresskalender über Fort- und Weiterbildungsangebote im pflegerischen Bereich. Abgerundet wird die Seite durch weiter führende Informationsangebote, wie Buchtipps, Fachzeitschriften und ein Glossar medizinischer Fachbegriffe. Schließlich stehen Download-Materialien, wie Ernährungstipps für Patienten während einer (immun)onkologischen Therapie zur Verfügung. Die Website ist frei zugänglich; eine Registrierung ist nicht erforderlich und kann von jedem Endgerät, wie Desktop, Tablet oder Smartphone aufgerufen werden. Mit diesem neuen Angebot setzt Bristol-Myers Squibb sein lange bestehendes Engagement für die Fortbildung von Pflegekräften fort. Bereits in der Vergangenheit war das forschende Pharmaunternehmen auf diesem Gebiet sehr aktiv und mit Ständen und Symposien auf Veranstaltungen für Pflegekräfte vertreten. Auch zukünftig wird BristolMyers Squibb, als Partner für die Pflege, sein Engagement fortsetzen. (reck)

\section{Arzneimittelgabe}

\section{Den Überblick behalten: Medikation im Alter}

» Ältere Menschen nehmen oft verschiedene Medikamente ein. Im Rahmen einer individualisierten Therapie sollte regelmäßig überprüft werden, ob die Medikation tatsächlich angemessen ist. Vor rund zwei Jahren wurde das Projekt InTherAKT zur Verbesserung der medikamentösen Versorgung in Altenhilfeeinrichtungen gestartet. Herzstück der gemeinsamen Arbeit von 15 Hausärzten, zwölf Apothekern und dem Pflegepersonal an zehn Altenhilfeeinrichtungen im Raum Münster ist eine Online-Plattform, die inzwischen zum Patent angemeldet wurde. Seit Anfang 2016 wurde es an rund 90 Patienten überprüft und optimiert. Wie der Projektleiter Prof. Jürgen Osterbrink, Vorstand des Instituts für Pflegewissenschaft und -praxis an der Paracelsus Medizinischen Privatuniversität, Salzburg, zum Hintergrund erläuterte, seien betagte Menschen besonders häufig von unerwünschten Arzneimittelinteraktionen betroffen. Vielfach seien die Interaktionen das Resultat nicht abgestimmter Prozesse der Leistungserbringer. Ziel des Projektes InTherAKT besteht deshalb darin, die Kommunikation und Kooperation zwischen den Berufsgruppen zu optimieren.

$\mathrm{Zu}$ den gravierendsten Folgen unerwünschter Arzneimittelwirkungen im Alter zählen aus Sicht von Apl. Prof. Georg Hempel, Institut fürPharmazeutische und Medizinische Chemie der Westfälischen Wilhelms-Universität, Münster, Gangunsicherheit, Synkopen und Stürze, die nicht selten lange
Krankenhausaufenthalte und den Verlust der Autonomie nach sich ziehen. In InTherAKT wird die Angemessenheit der Medikation anhand des „Medication Appropriateness Index“ (MAI) erhoben - und hier ergab sich eine Verbesserung um durchschnittlich $25 \%$. Besonders deutlich zeigte sich der Erfolg bei Patienten, die vorher sehr viele Medikamente einnehmen mussten. Im Einzelfall konnte die Zahl der Medikamente um bis zu fünf Präparate reduziert werden.

(he)

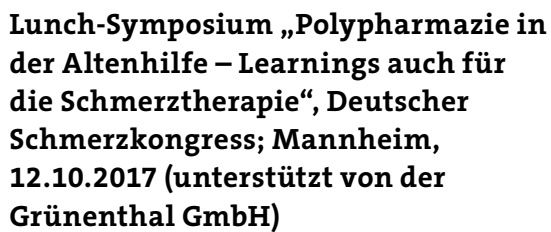

